\title{
Effect of sericin on the p38MAPK signaling pathway and NLRP3 inflammasome in the kidney of type 2 diabetic rats
}

\author{
DONGHUI LIU ${ }^{1}, \mathrm{CHENG}_{\mathrm{CHEN}}{ }^{2}$, DANDAN WANG $^{3}, \mathrm{ZHIHONG} \mathrm{CHEN}^{1}$ and CHENGJUN SONG ${ }^{1}$ \\ Departments of ${ }^{1}$ Human Anatomy and ${ }^{2}$ Physiology, Chengde Medical University, Chengde, Hebei 067000; \\ ${ }^{3}$ Department of Anatomy, Shijiazhuang Medical College, Shijiazhuang, Hebei 050599, P.R. China
}

Received February 25, 2020; Accepted July 31, 2020

DOI: $10.3892 /$ etm.2020.9397

\begin{abstract}
The present study aimed to investigate the effects of sericin on the p38MAPK signaling pathway and nucleotidebinding oligomerization domain-like receptor protein 3 (NLRP3) inflammasome in the kidney of rats with type 2 diabetes mellitus (T2DM). A total of 36 male Sprague-Dawley rats were randomly divided into the normal group, T2DM model group and sericin group $(n=12$ rats/group). A T2DM model was developed through intraperitoneal injection of streptozotocin (35 $\mathrm{mg} \cdot \mathrm{kg}^{-1} \cdot \mathrm{d}^{-1}$ for 2 consecutive days), and a high-fat and high-sugar diet. The T2DM rats in the sericin group were administered $2.4 \mathrm{~g} \cdot \mathrm{kg}^{-1} \cdot \mathrm{d}^{-1}$ sericin for 35 days, and rats in the other groups were administered an equal volume of normal saline for 35 days. Fasting blood glucose was measured using the glucose oxidase method. Kidney tissue morphology was observed by H\&E staining. Immunohistochemistry, western blotting, ELISA and reverse transcription-quantitative PCR were used to detect the levels of MKK6, p38MAPK, phosphorylated (p)-p38MAPK, NF- $\kappa$, IL-1 $\beta$, IL-6, NLRP3 and caspase- 1 in rat kidney tissues. The results revealed that blood glucose concentration, and the expression levels of MKK6, p-p38MAPK, NF- $\mathrm{B}$, IL-1 $\beta$, IL-6, NLRP3 and caspase-1 were significantly increased in the T2DM group compared with those in the normal group $(\mathrm{P}<0.05)$. In addition, obvious pathological changes were observed in the T2DM group. Conversely, glucose concentration, and the expression levels of MKK6, p-p38MAPK, NF- $\kappa$ B, IL-1 $\beta$, IL-6, NLRP3 and caspase-1 were significantly reduced in the sericin group compared with those in the T2DM group $(\mathrm{P}<0.05)$. The
\end{abstract}

Correspondence to: Dr Zhihong Chen or Mr. Chengjun Song, Department of Human Anatomy, Chengde Medical University, Anyuan Road, Shuangqiao, Chengde, Hebei 067000, P.R. China

E-mail: czh1971@126.com; 313804850@qq.com

E-mail: songchengjun@126.com

Abbreviations: DN, diabetic nephropathy; NLRP3, nucleotide-binding oligomerization domain-like receptor protein 3; T2DM, type 2 diabetes mellitus

Key words: sericin, T2DM, kidney, p38MAPK signaling pathway, NLRP3 inflammasome pathological changes were also obviously reduced. Notably, there was no significant difference in p38MAPK expression among the three groups $(\mathrm{P}>0.05)$. Collectively, the present study revealed that sericin may downregulate the expression

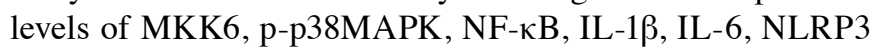
and caspase-1, and inhibit activation of renal p38MAPK signaling and NLRP3-associated inflammation, which in turn may protect against kidney damage caused by T2DM.

\section{Introduction}

Diabetic nephropathy (DN) is one of the most common and serious chronic complications of diabetes. Without appropriate treatment, DN can progress to end-stage renal disease $(1,2)$; however, the pathogenesis of $\mathrm{DN}$ is complex and unclear. Previous studies have revealed that $\mathrm{DN}$ is associated with genetic susceptibility, abnormal renal hemodynamics, oxidative stress and inflammatory response (3-5). The p38MAPK signaling pathway is an important branch of the MAPK pathway, which is activated in the kidney, and can promote glomerulosclerosis and tubulointerstitial fibrosis by participating in inflammatory reactions and oxidative stress (6). Nucleotide-binding oligomerization domain-like receptor protein 3 (NLRP3) inflammasome is a molecular platform that activates caspase-1. The NLRP3 inflammasome can regulate the maturation and secretion of pro-inflammatory cytokines, and can thus regulate the chronic inflammatory response $(7,8)$. Sustained hyperglycemia in type 2 diabetes mellitus (T2DM) can activate the p38MAPK signaling pathway and the NLRP3 inflammasome, which can further affect the occurrence and development of DN by regulating the expression of downstream related factors (9-11) Therefore, studying changes in the p38MAPK signaling pathway and NLRP3 inflammasome in the kidneys of patients with diabetes may be of great significance for further study of the pathogenesis of DN.

At present, the clinical treatment of T2DM includes improving diet, appropriate exercise, drug control of blood glucose and surgery (12-14). Most of these drugs can control blood glucose well, but long-term use is prone to drug resistance and adverse reactions $(3,14)$. For example, oral hypoglycemic drugs such as sulfonylureas, biguanides and insulin sensitizers can cause drowsiness and gastrointestinal reactions (13). After subcutaneous injection, allergic reactions, sarcoidosis and blood stasis may occur. In addition, it may 
cause lipoatrophy under the skin, which will lead to unstable insulin absorption, and it is very difficult to resolve spontaneously once it occurs (14). Therefore, it is necessary to identify a drug with reliable therapeutic effect, fewer adverse reactions and good kidney protection.

Sericin is a water extract obtained from the cocoon of silkworms (Bombyx mori) and is a natural water-soluble protein. It has been demonstrated that sericin can effectively reduce blood glucose in rats with $\mathrm{T} 2 \mathrm{DM}$ and protect against diabetes-induced kidney damage $(15,16)$. However, the underlying mechanism is currently unclear. The present study aimed to investigate the protective effects and underlying mechanism of sericin on kidney injury in rats with T2DM. Changes in key proteins associated with the p38MAPK signaling pathway and NLRP3 inflammasome were analyzed. The present findings may provide novel insights for the prevention and treatment of T2DM and its complications.

\section{Materials and methods}

Animals. Male Sprague-Dawley rats ( $\mathrm{n}=42$; weight, 170-190 g; age, 5-6 weeks) were purchased from Beijing Vital River Laboratory Animal Technology Co., Ltd. and were maintained under standard conditions (Temperature, $20-24^{\circ} \mathrm{C}$; humidity, 50-55\%; free access to food and water; $12 / 12 \mathrm{~h}$ light/dark cycle). The health and behaviors of the rats were monitored every day. The main humane endpoints of reduced heart rate and respiration rate were used to determine when animals should be euthanized. All animal experimental procedures were approved by the Ethics Committee of Chengde Medical University (Chengde, China).

Establishment of a rat model of T2DM. A rat model of T2DM was established in 30 rats according to previous studies (17-19). Briefly, rats were fed a high-fat and high-sugar diet (59\% of basic feed, $20 \%$ of white sugar, $3 \%$ of egg yolk, $18 \%$ of lard) for 4 weeks $(20,21)$, and were then intraperitoneally injected with $35 \mathrm{mg} \cdot \mathrm{kg}^{-1} \cdot \mathrm{d}^{-1}$ streptozotocin (Sigma-Aldrich; Merck KGaA) for 2 days. If fasting blood glucose was $\geq 11.1 \mathrm{mmol} \cdot \mathrm{l}^{-1}$ for three consecutive measurements at $72 \mathrm{~h}$ after the last injection, the T2DM model was considered successfully established. During model establishment, six rats died (22-24).

Animal grouping and treatment. After successful T2DM modeling, the rats continued to be fed with a high-fat and high-sugar diet for 4 weeks (17). Subsequently, the T2DM model rats were randomly divided into the T2DM model group and the sericin group ( $\mathrm{n}=12$ rats/group). Rats in the sericin and T2DM model groups were administered $2.4 \mathrm{~g} \cdot \mathrm{kg}^{-1} \cdot \mathrm{d}^{-1}$ sericin by gavage and an equal volume of normal saline, respectively, for 35 days. In parallel, rats in the normal group (fed with a normal diet ad libitum; $\mathrm{n}=12$ ) were administered an equal volume of normal saline for 35 days. After treatment for 35 days, the rats in each group were fasted for $12 \mathrm{~h}$. Blood samples were collected via the tail vein for blood glucose testing once a week; 5-10 $\mu \mathrm{l}$ blood was collected each time. Kidney tissues were collected following anesthesia (intraperitoneal injection of $300 \mathrm{mg} / \mathrm{kg} 10 \%$ chloral hydrate) and sacrifice by decapitation. During anesthesia, the rats did not exhibit any signs of peritonitis, pain or discomfort.
Detection of blood glucose levels. Blood samples from each group of rats were centrifuged at $800 \times \mathrm{g}$ for $20 \mathrm{~min}$ at $4^{\circ} \mathrm{C}$. The serum was then collected and glucose levels were detected using the Beckman Coulter AU5800 Automatic Biochemical Analyzer (Beckman Coulter, Inc.).

$H \& E$ staining. The kidney morphology of each group was observed by H\&E staining. Briefly, kidney samples were fixed in Bouins fixative liquid [a mixture of picric acid saturated liquid (1.22\%), formaldehyde and glacial acetic acid in a ratio of 15:5:1] at room temperature for $24 \mathrm{~h}$, embedded in paraffin and cut into $5-\mu \mathrm{m}$ sections. The sections were stained with hematoxylin (room temperature for $7 \mathrm{~min}$ ), eosin (room temperature for $1 \mathrm{~min}$ ), dehydrated in gradient ethanol and incubated with xylene (room temperature for $30 \mathrm{~min}$ ). The samples were then observed under a light microscope (OLYMPUS BH-2; Olympus Corporation).

Immunohistochemistry. Kidney tissue sections were incubated with $3 \% \mathrm{H}_{2} \mathrm{O}_{2}$-methanol for $30 \mathrm{~min}$ at $37^{\circ} \mathrm{C}$ to inactivate endogenous peroxidase activity. Antigen retrieval was then performed in a microwave for $8 \mathrm{~min}$ with $0.01 \mathrm{M}$ citrate buffer (pH, 6.0). Subsequently, tissue sections were incubated with the following primary antibodies at $4^{\circ} \mathrm{C}$ overnight: MKK6 (1:100; cat. no. A2575; Wuhan Aibotek Biotechnology Co., Ltd.), phosphorylated (p)-p38MAPK (1:50; cat. no. sc-166182; Santa Cruz Biotechnology, Inc.), NF-кB (1:50; cat. no. ab16502; Abcam), NLRP3 (1:100; cat. no. DF7438; Affinity Biosciences), caspase-1 (1:200; cat. no. A0964; Wuhan Aibotek Biotechnology Co., Ltd.), IL-1 $\beta$ (1:200; cat. no. AF5103; Affinity Biosciences) and IL-6 (1:200; cat. no. DF6087; Affinity Biosciences). After washing with PBS, secondary antibodies (1:300; Biotin labeled goat anti-rabbit IgG polymer; cat. no. SP-9001; Beijing Zhong Shan-Golden Bridge Biological Technology Co., Ltd.) were added and incubated at $37^{\circ} \mathrm{C}$ for $40 \mathrm{~min}$. After DAB staining at room temperature for $2 \mathrm{~min}$, hematoxylin counterstaining was performed at room temperature for $3 \mathrm{~min}$. Brownish yellow and/or tan particles in the glomeruli and/or renal tubules were considered positive staining. Six rats were randomly selected from each group, and three non-continuous kidney slices were randomly selected from each rat. Three non-overlapping fields containing glomeruli and tubules were randomly selected for observation in each slice. The integrated optical density value of the positive staining in each visual field was calculated using Image-ProPlus 6.0 software (Media Cybernetics, Inc.), and the average optical density value was taken as the relative expression level of the target protein.

Western blotting. Total protein was extracted from kidney tissues using the RIPA (cat. no. R0020, Beijing Soleibao Technology Co., Ltd.) and quantified using the BCA protein concentration determination kit (cat.no.PC0020,Beijing Soleibao Technology Co., Ltd.). Total protein $(60 \mu \mathrm{g})$ was separated by SDS-PAGE on $10-12 \%$ gels and was then transferred onto PVDF membranes (cat. no. IEVH10100; EMD Millipore). After blocking with $5 \%$ non-fat milk at room temperature for $1 \mathrm{~h}$, membranes were incubated with the following primary antibodies at room temperature for $2 \mathrm{~h}$ : MKK6 (1:500; cat. no. A2575; Wuhan Aibotek Biotechnology Co., Ltd.), p38MAPK 
Table I. Primers used in this study.

\begin{tabular}{|c|c|c|}
\hline Gene & Sequence $\left(5^{\prime}-3^{\prime}\right)$ & Primer length (bp) \\
\hline MKK6 & $\begin{array}{l}\text { F: AACGGCCCACGTATCCAGAG } \\
\text { R: CCACCAATCCACAGTAGGGTCA }\end{array}$ & 147 \\
\hline p38MAPK & $\begin{array}{l}\text { F: TTACCGATGACCACGTTCAGTTTC } \\
\text { R: AGCGAGGTTGCTGGGCTTTA }\end{array}$ & 107 \\
\hline $\mathrm{NF}-\kappa \mathrm{B}$ & $\begin{array}{l}\text { F: GATGGGACGACACCTCTACACATA } \\
\text { R: CCCAAGAGTCGTCCAGGTCA }\end{array}$ & 130 \\
\hline IL-1 $\beta$ & $\begin{array}{l}\text { F: CCCTGAACTCAACTGTGAAATAGCA } \\
\text { R: CCCAAGTCAAGGGCTTGGAA }\end{array}$ & 111 \\
\hline IL-6 & $\begin{array}{l}\text { F: ATTGTATGAACAGCGATGATGCAC } \\
\text { R: CCAGGTAGAAACGGAACTCCAGA }\end{array}$ & 150 \\
\hline NLRP3 & $\begin{array}{l}\text { F: CTGAAGCATCTGCTCTGCAACC } \\
\text { R: AACCAATGCGAGATCCTGACAAC }\end{array}$ & 87 \\
\hline Caspase-1 & $\begin{array}{l}\text { F: ACTCGTACACGTCTTGCCCTCA } \\
\text { R: CTGGGCAGGCAGCAAATTC }\end{array}$ & 190 \\
\hline GAPDH & $\begin{array}{l}\text { F: GGCACAGTCAAGGCTGAGAATG } \\
\text { R: ATGGTGGTGAAGACGCCAGTA }\end{array}$ & 143 \\
\hline
\end{tabular}

F, forward; R, reverse; NLRP3, nucleotide-binding oligomerization domain-like receptor protein 3.

(1:500; cat. no. AF6456; Affinity Biosciences), p-p38MAPK (1:1,000; cat. no. sc-166182; Santa Cruz Biotechnology, Inc.), NF-кB (1:2,000; cat. no. ab16502; Abcam), NLRP3 (1:1,000; cat. no. DF7438; Affinity Biosciences), caspase -1 (1:500; cat. no. A0964; Wuhan Aibotek Biotechnology Co., Ltd.) and $\beta$-actin (1:500; cat. no. AF7018; Affinity Biosciences). The secondary antibody (1:10,000; cat. no. 074-1806; KPL, Inc.) was then added and the membranes were incubated at room temperature for $1.5 \mathrm{~h}$. Subsequently, the membranes were washed and subjected to color development using the Super ECL Plus ultra-sensitive luminescent solution (cat. no. P1050; Beijing Pulilai Gene Technology Company). The gray intensities of each band were measured using Quantity One-v 4.6.2 software (Bio-Rad Laboratories, Inc.). The ratio of the gray value of the target protein to $\beta$-actin was taken as the relative expression level of each target protein.

ELISA.The detection of IL-1 $\beta$ and IL-6 in serum was performed using the Rat IL-1 $\beta /$ IL-6 ELISA kit (RayBiotech, Inc.; IL-1 $\beta$, cat. no. Q63264; IL-6 cat. no. P20607), according to the manufacturer's protocol. The absorbance was measured at $450 \mathrm{~nm}$ using a microplate reader (ELX808; BioTek Corporation). The corresponding concentration was then calculated.

Reverse transcription-quantitative PCR (RT-qPCR). Total RNA was extracted from $100 \mathrm{mg}$ kidney tissue using the TaKaRa MiniBEST Universal RNA Extraction kit (cat. no. 9767; Takara Biotechnology Co., Ltd.). cDNA was then generated by using the PrimeScript ${ }^{\mathrm{TM}}$ RT Master Mix (Takara Biotechnology Co., Ltd.; cat. no. RR036A) at $37^{\circ} \mathrm{C}$ for $15 \mathrm{~min}$, and $85^{\circ} \mathrm{C}$ for $5 \mathrm{sec}$. The mRNA expression levels of MKK6, p38MAPK, NF- $\mathrm{B}$, IL-1 $\beta$, IL-6, NLRP3, caspase-1 and GAPDH genes were detected with RT-qPCR. The primer sequences are listed in Table I. qPCR was performed using 12.5 $\mu \mathrm{l}$ SYBR Premix Ex TaqII (Takara Biotechnology Co., Ltd.; cat. no. RR820A), $1 \mu$ 1 PCR forward primer, $1 \mu \mathrm{l}$ PCR reverse primer, $2 \mu \mathrm{l}$ cDNA and $8.5 \mu \mathrm{l}$ $\mathrm{ddH}_{2} \mathrm{O}$. The thermocycling conditions were as follows: $95^{\circ} \mathrm{C}$ for $30 \mathrm{sec}$, followed by 40 cycles at $95^{\circ} \mathrm{C}$ for $5 \mathrm{sec}$ and $60^{\circ} \mathrm{C}$ for $30 \mathrm{sec}$, and a final step at $60^{\circ} \mathrm{C}$ for $5 \mathrm{~min}$. The relative expression level of each target gene was analyzed using the $2^{-\Delta \Delta \mathrm{Cq}}$ method (25).

Statistical analysis. Data were analyzed using IBM SPSS Statistics 21.0 statistical software (IBM Corp.). Data are presented as the mean \pm SD of three independent experiments. Multi-group comparisons were performed by one-way ANOVA and pairwise comparisons were conducted using LSD-t post hoc test. $\mathrm{P}<0.05$ was considered to indicate a statistically significant difference.

\section{Results}

Sericin reduces blood glucose levels in rats. To investigate the effects of sericin on blood glucose levels, blood glucose was measured using the glucose oxidase method. The blood glucose of rats in the normal, T2DM model and sericin groups was $10.83 \pm 2.03,29.45 \pm 4.82$ and $13.20 \pm 4.09 \mathrm{mmol} \cdot \mathrm{l}^{-1}$, respectively (Table II). The blood glucose levels in the T2DM model group were significantly higher than those in the normal group $(\mathrm{P}<0.05)$, whereas those in the sericin group were significantly lower than those in the T2DM model group $(\mathrm{P}<0.05)$.

Sericin relieves the T2DM-induced pathological changes to the rat kidney. To further address the effects of sericin, H\&E staining was conducted to detect the morphological structure of rat kidneys in each group. Compared with in rats in the normal group, renal glomerular hypertrophy and mesangial hyperplasia, 
Table II. Blood glucose levels of each group.

\begin{tabular}{|c|c|c|c|}
\hline & Normal group & T2DM model group & Sericin groups \\
\hline Blood glucose $\left(\mathrm{mmol} \cdot \mathrm{l}^{-1}\right)$ & $10.83 \pm 2.03$ & $29.45 \pm 4.82^{\mathrm{a}}$ & $13.20 \pm 4.09^{\mathrm{b}}$ \\
\hline
\end{tabular}
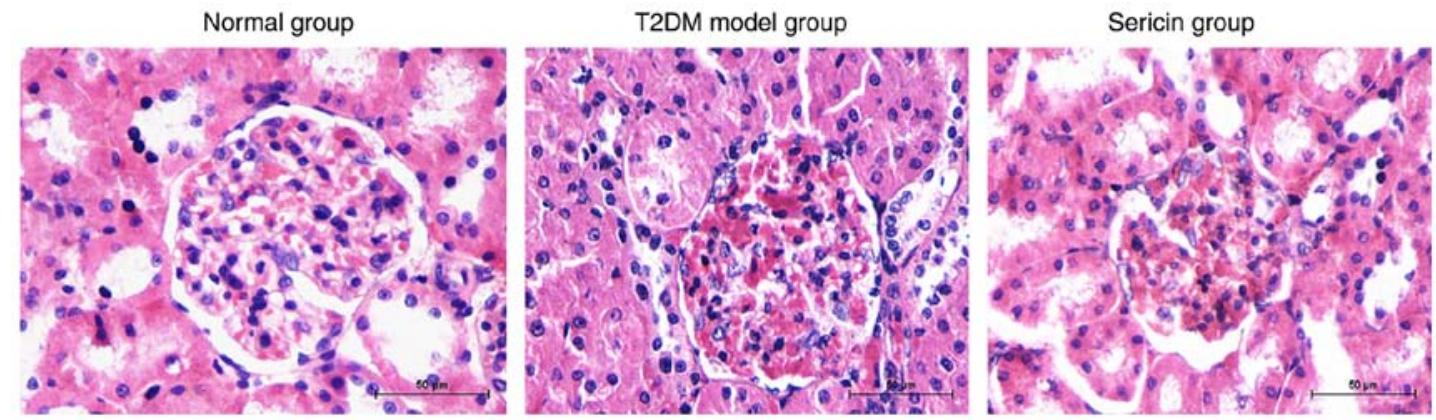

Figure 1. Morphological structure of the rat kidney in each group. H\&E staining was performed to observe the pathological changes in the normal, T2DM model and sericin groups. Black arrows: Mesangial matrix; Green arrows: Mesangial cells; Red arrows: Basement mem brane. Scale bar, $50 \mu \mathrm{m}$. T2DM, type 2 diabetes mellitus.

thickened basement membrane and extracellular matrix accumulation were detected in the T2DM model group (Fig. 1). The renal pathological manifestations in the sericin group were obviously reduced. The renal mesangial hyperplasia, basement membrane thickening and renal interstitial fibrosis were milder in the sericin group than those in the T2DM model group.

Sericin suppresses the expression of p38MAPK signaling pathway-related proteins and genes in the rat kidney. The present study measured the effects of sericin on the expression levels of $\mathrm{p} 38 \mathrm{MAPK}$ signaling pathway-related proteins. The positive staining of MKK6, p-p38MAPK, NF- $\kappa \mathrm{B}$, IL-1 $\beta$ and IL-6 protein was shown as brownish yellow and/or brown granules, which were mainly distributed in renal tubular epithelial cells and mesangial cells (Fig. 2A). The protein expression levels of MKK6, p-p38MAPK, NF- $\kappa$ B, IL-1 $\beta$ and IL-6 were highest in the T2DM model group and lowest in the normal group (Fig. 2B). The results of western blotting are shown in Fig. 3A. Clear MKK6, p38MAPK, p-p38MAPK, $\mathrm{NF}-\kappa \mathrm{B}$ and $\beta$-actin bands were observed at $37,43,43,45$ and $42 \mathrm{kDa}$, respectively. The protein expression levels of MKK6, p-p38MAPK and NF- $\kappa$ B were highest in the T2DM model group and lowest in the normal group (Fig. 3B). Levels of IL-1 $\beta$ and IL-6 were further analyzed using ELISA (Fig. 3C). The levels of IL-1 $\beta$ and IL- 6 in the kidney tissue of the T2DM model group were significantly higher than that of the normal group $(\mathrm{P}<0.05)$; while those in the sericin group were significantly lower than that of the T2DM model group $(\mathrm{P}<0.05)$. The mRNA expression levels of MKK6, p38MAPK, NF- $\kappa \mathrm{B}$, IL- $1 \beta$ and IL- 6 were measured using RT-qPCR (Fig. 3D). Statistically, the protein and mRNA expression levels of MKK6, NF- $\kappa \mathrm{B}, \mathrm{IL}-1 \beta$ and IL- 6 in the kidneys of the T2DM model group were significantly higher than those in the normal group $(\mathrm{P}<0.05)$. Notably, the protein and mRNA expression levels of MKK- $6, N F-\kappa B$, IL- $1 \beta$ and IL- 6 were significantly lower in the sericin group compared with those in the T2DM model group $(\mathrm{P}<0.05)$. The protein expression levels of p-p38MAPK were significantly higher in the kidney of rats in the T2DM model group than those in the normal group $(\mathrm{P}<0.05)$, whereas the protein expression levels of p-p38MAPK were significantly lower in the kidney of rats the sericin group compared with those in the T2DM model group $(\mathrm{P}<0.05)$. However, there was no significant difference in the mRNA and protein expression levels of p38MAPK in each group of rats $(\mathrm{P}>0.05)$. Taken together, these results indicated that sericin reduced the expression of p38MAPK signaling pathway-related genes and proteins in rat kidney samples.

Sericin reduces the expression of NLRP3 and caspase-1 in the kidneys of rats. The present study further tested the effects of sericin on the expression levels of NLRP3 and caspase-1. Using immunohistochemistry, NLRP3 and caspase-1 proteins were shown as brownish yellow and/or tan granules. NLRP3 protein was mainly expressed in the cytoplasm of renal tubular epithelial cells, and caspase- 1 protein was mainly expressed in renal tubular epithelial cells and mesangial cells (Fig. 4A). The protein expression levels of NLRP3 and caspase-1 were highest in the T2DM model group and lowest in the normal group (Fig. 4B). The results of western blotting are shown in Fig. 5A. Clear NLRP3, caspase- 1 and $\beta$-actin bands were seen at 118, 45 and $42 \mathrm{kDa}$, respectively. Consistently, the protein expression levels of NLRP3 and caspase-1 were highest in the T2DM model group and lowest in the normal group (Fig. 5B). RT-qPCR was conducted to detect their expression at the mRNA level (Fig. 5C). The results showed that the protein and mRNA expression levels of NLRP3 and caspase-1 were significantly higher in the kidney of rats in the T2DM model group than those in the normal group $(\mathrm{P}<0.05)$. Conversely, their expression levels were significantly lower in the kidney of rats in the sericin group compared with those in the T2DM model group $(\mathrm{P}<0.05)$. These results indicated that sericin may reduce the expression of NLRP3 and caspase-1 in the kidneys of rats. 

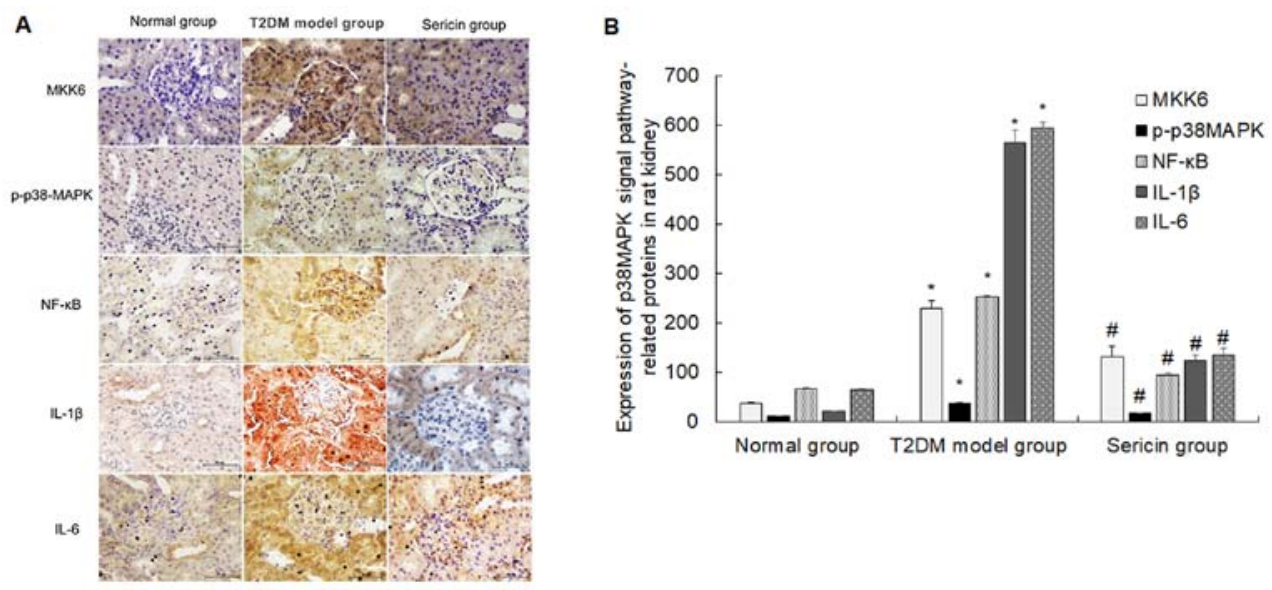

Figure 2.p38MAPK signal pathway-related protein expression in the rat kidney of each group was detected by immunohistochemical staining. (A) Representative images of the normal, T2DM model and sericin groups are shown. Scale bar, $50 \mu \mathrm{m}$. (B) Relative staining intensities were calculated. " $\mathrm{P}<0.05 \mathrm{vs}$. the normal group; ${ }^{~} \mathrm{P}<0.05$ vs. the T2DM model group. T2DM, type 2 diabetes mellitus; $\mathrm{p}$-, phosphorylated.
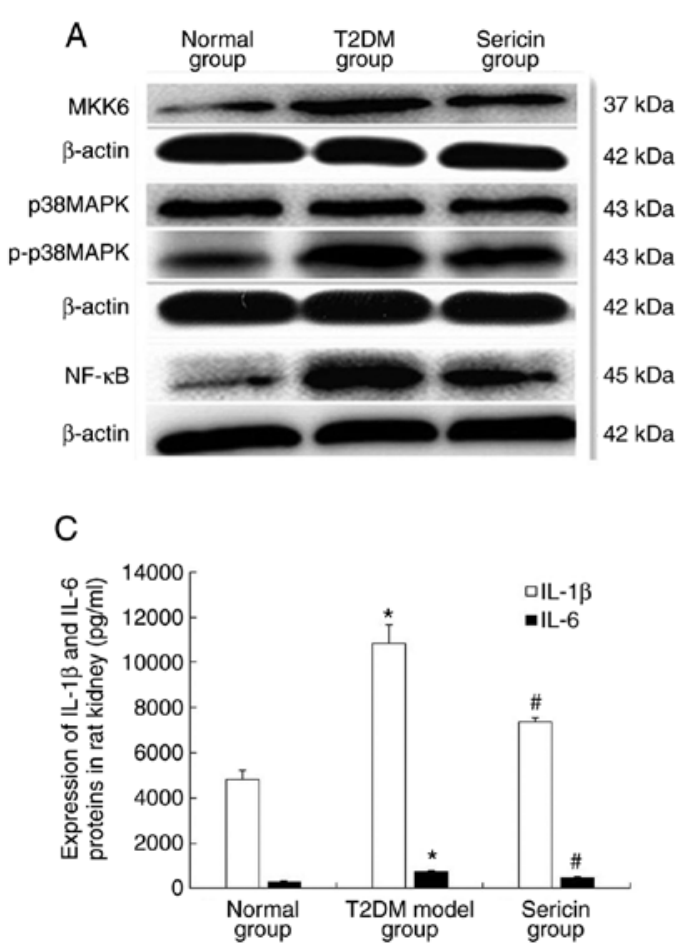

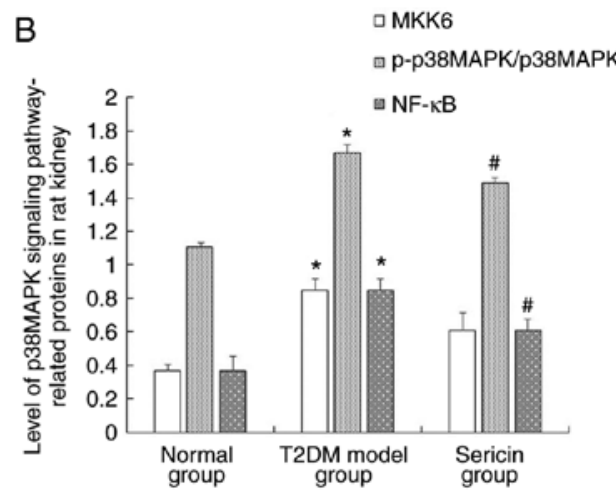

D

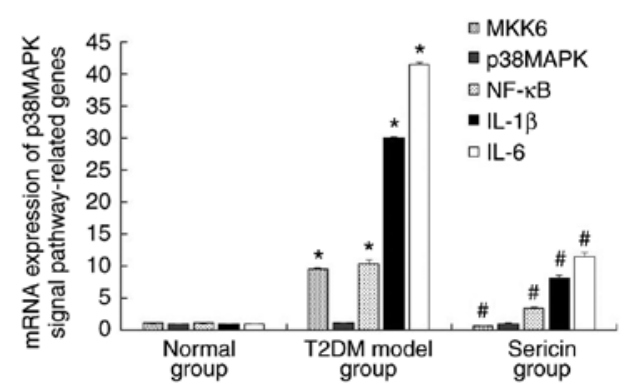

Figure 3. Expression of p38MAPK signaling pathway-related proteins and genes, and IL-1 $\beta$ and IL-6 in the rat kidney of each group. (A) Representative western blotting results of p38MAPK signaling pathway-related proteins in the normal, T2DM model and sericin groups are shown. (B) Relative protein expression levels were calculated. (C) ELISA was used to detect protein levels of IL-1 $\beta$ and IL-6. (D) Expression of p38MAPK signal pathway-related mRNA was detected by reverse transcription-quantitative $\mathrm{PCR}$. " $\mathrm{P}<0.05$ vs. the normal group; ${ }^{\sharp} \mathrm{P}<0.05$, vs. the T2DM model group. T2DM, type 2 diabetes mellitus; $\mathrm{p}$-, phosphorylated.

\section{Discussion}

T2DM is a chronic metabolic disease characterized by insulin resistance and multiple organ damage, which often occurs in the kidneys and can cause DN. DN is one of the serious microvascular complications of T2DM $(26,27)$. Typical pathological changes of DN include hypertrophy of renal cells, thickening of the glomerular basement membrane and accumulation of the extracellular matrix, which can further cause glomerulosclerosis and tubulointerstitial fibrosis, and eventually lead to renal insufficiency or renal failure $(28,29)$. The pathogenesis of DN has not yet been fully elucidated, but scholars have recognized that it is caused by the participation of multiple risk factors in a certain genetic background, and the inflammatory response is known to have an important role $(30,31)$.

The p38MAPK signaling pathway can regulate the inflammatory response of the kidney and is one of target pathways of anti-inflammatory drugs (32). The p38MAPK signaling pathway is characterized by a three-level kinase cascade; under the action of hyperglycemia, MAPK kinase is phosphorylated first, and then the threonine-tyrosine site in the p38MAPK domain is phosphorylated to activate p38MAPK by activating MKK6, which becomes p-p38MAPK $(6,33)$. The biological activity of p-p38MAPK further induces $N F-\kappa B$, 
A

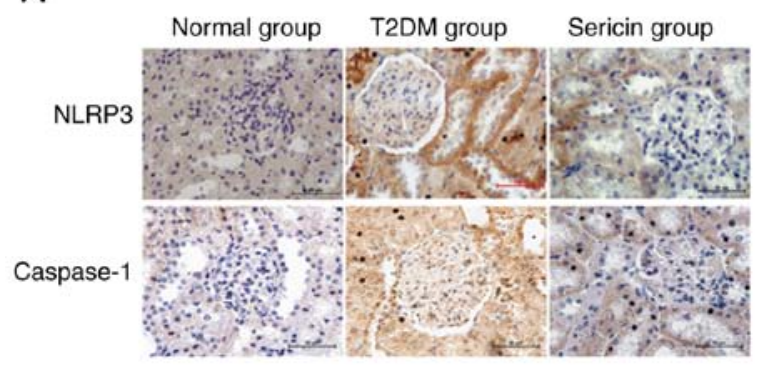

B

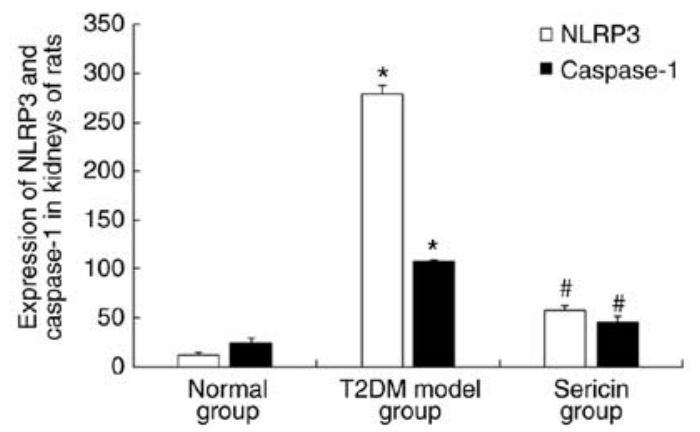

Figure 4. Expression of NLRP3 and caspase-1 in the kidney of rats from each group were detected by immunohistochemical staining. (A) Representative images of the normal, T2DM model and sericin groups are shown. Scale bar, $50 \mu \mathrm{m}$. (B) Relative staining intensities were calculated. "P<0.05 vs. the normal group; ${ }^{\#} \mathrm{P}<0.05$, vs. the T2DM model group. T2DM, type 2 diabetes mellitus; NLRP3, nucleotide-binding oligomerization domain-like receptor protein 3.
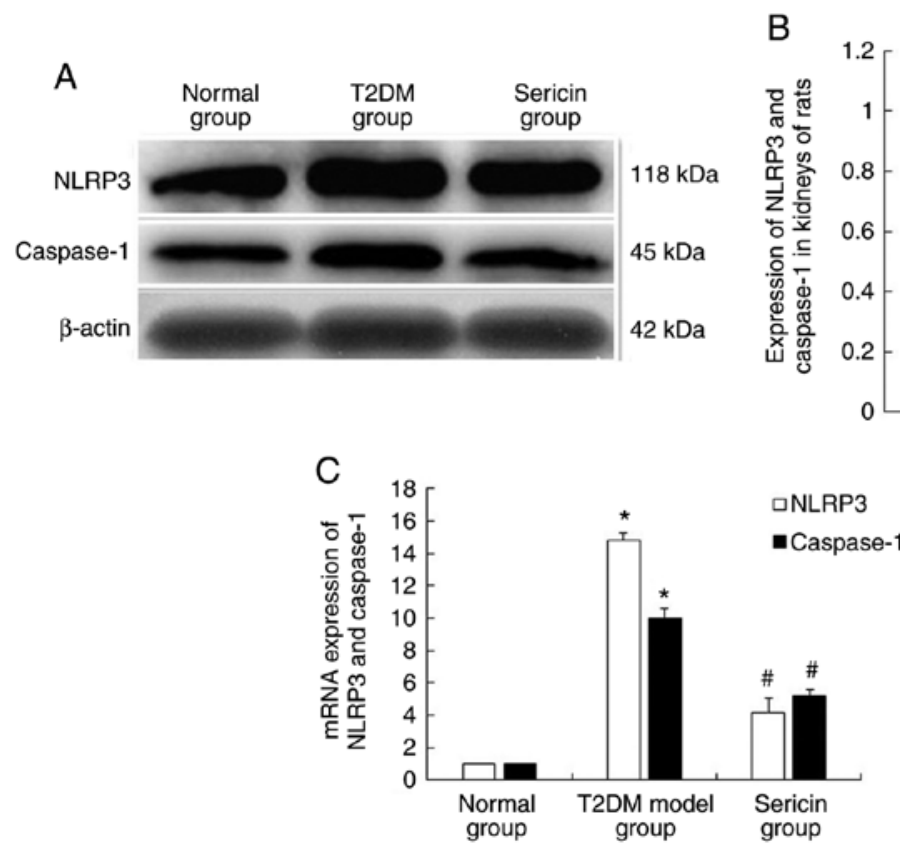

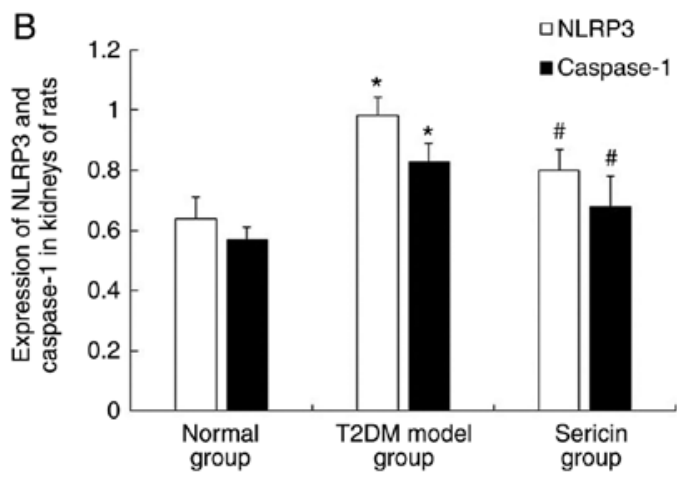

LRP3 group

Figure 5. Expression of NLRP3 and caspase-1 in the kidney of rats from each group. (A) Representative western blotting results of NLRP3 and caspase-1 in the normal, T2DM model and sericin groups are shown. (B) Relative protein expression levels were calculated. (C) mRNA expression levels of NLRP3 and caspase- 1 were detected by reverse transcription-quantitative PCR. ${ }^{*} \mathrm{P}<0.05$ vs. the normal group; ${ }^{\#} \mathrm{P}<0.05$, vs. the T2DM model group. T2DM, type 2 diabetes mellitus; NLRP3, nucleotide-binding oligomerization domain-like receptor protein 3.

allowing it to enter the nucleus from the cytoplasm, where it binds to the target gene promoter or enhancer to activate IL-1 $\beta$ and IL-6, thus inducing inflammation $(34,35)$. IL-1 $\beta$ and IL-6 are important regulators of inflammation. IL-1 $\beta$ can stimulate the proliferation of mesangial cells and release reactive oxygen to participate in kidney inflammation $(36,37)$. IL-6 is involved in the inflammatory response of the kidney through promoting mesangial cell proliferation, extracellular matrix synthesis and local infiltration of macrophages in renal tissues, and can accelerate glomerular sclerosis, lead to tubular atrophy and interstitial fibrosis, and thereby advance the progression of DN $(37,38)$. The present study revealed that fasting blood glucose, and the expression levels of renal MKK6, p-p38MAPK, NF- $\mathrm{B}$, IL-1 $\beta$ and IL-6 were significantly higher in the T2DM model group compared with those in the normal group. These findings indicated that under hyperglycemia, the p38MAPK signaling pathway was activated in the rat kidney, which may induce the activation of downstream inflammatory cells and promote the expression of inflammatory mediators. This may therefore lead to an increase in the production of inflammatory factors that affect the kidney tissue, and cause changes in kidney morphology and function. In addition, hyperglycemia may stimulate the renal p38MAPK signaling pathway to further induce oxidative stress and accelerate the process of renal fibrosis, whereas oxidative stress can also activate the p38MAPK signaling pathway. This vicious cycle may further promote the occurrence and development of DN $(34,39)$. Notably, p38MAPK is biologically active only in the form of p-p38MAPK; therefore, no significant difference was detected in p38MAPK expression between the groups of rats in the present study.

The NLRP3 inflammasome is an important part of the immune inflammatory response and has been reported to serve an important role in the occurrence and development 
of DN (40). The NLRP3 inflammasome consists of NLRP3, caspase-1 and apoptosis-related speckle-like protein. Under the stimulation of hyperglycemia, the domain of NLRP3 is changed and the caspase-1 precursor is cleaved to generate active caspase- 1 with hydrolase activity. Activated caspase-1 can promote the processing of inactive IL-1 $\beta$ precursors, and also induce the maturation and secretion of IL-1 $\beta$ to participate in the inflammation of the kidney (41-43). Notably, elevated IL-1 $\beta$ levels can also activate NF- $\kappa B$ in renal tubular epithelial cells, further amplifying the inflammatory response and accelerating the process of renal fibrosis (44). In the present study, the expression levels of NLRP3 and caspase-1 were significantly higher in the kidney of rats in the T2DM model group compared with those in the normal group, thus suggesting that under hyperglycemic conditions, the NLRP3 inflammasome may be activated, which could further aggravate the inflammatory response of the kidney, causing significant pathological changes in the kidneys of the T2DM model group rats.

$\mathrm{DN}$ is a fatal chronic complication of diabetes. Once it develops into end-stage renal disease, severe and complex metabolic disorders often occur, resulting in poor prognosis (45). Therefore, only with early targeted prevention and treatment of DN can further development of kidney damage be effectively controlled and delayed. At present, the treatment of clinical DN is mainly based on hypoglycemic, antihypertensive, lipid-lowering and anti-inflammatory therapies, and other measures (46). Although various hypoglycemic drugs can control blood glucose, long-term administration is prone to induce drug resistance, and liver and kidney damage. Therefore, a large number of studies have focused on natural drugs that have a reliable hypoglycemic effect and exhibit kidney protection (47-49). Sericin is a natural water-soluble protein found in silk cocoons, which is composed of 18 amino acids. In the molecular structure, hydroxyl, carboxyl and amino groups of amino acids with strong polar side groups account for the vast majority (50). Sericin is biodegradable and has no toxic side effects on the human body. In China, silkworm cocoons soaked in boiling water have been reported to reduce blood glucose (51). Our previous studies also demonstrated that sericin was able to lower blood glucose and protect organs, such as the liver, kidney and islet cells $(16,52,53)$; however, the specific underlying mechanism is still unclear. In the present study, the rats were treated with sericin by gavage. The levels of fasting blood glucose, and the expression levels of MKK6, p-p38MAPK, NF-кB, IL-1 $\beta$, IL-6, NLRP3 and caspase-1 in the kidney were lower in the sericin group than those in the T2DM model group. In addition, the pathological changes in the kidneys of the sericin group were markedly reduced. These findings indicated that the expression of related factors in the p38MAPK signaling pathway and NLRP3 inflammasome in the kidney of rats with T2DM changed following gavage treatment with sericin. Therefore, sericin may reduce blood glucose and renal injury in T2DM rats by regulating these factors.

The present study had some limitations. Firstly, there was a lack of experiments on the interaction between sericin and the p38MAPK signaling pathway in models without hyperglycemia. Secondly, the phosphorylation levels of related factors in the p38MAPK signaling pathway were not analyzed due to the limited amount of kidney tissues and the unavailability of the antibody. Further studies are thus warranted.
In conclusion, the present study demonstrated that sericin reduced blood glucose, and inhibited activation of the p38MAPK signaling pathway and NLRP3 inflammasome in the kidney of rats with T2DM. These effects may reduce inflammation, weaken T2DM-induced kidney damage, and delay the occurrence and development of DN.

\section{Acknowledgements}

Not applicable.

\section{Funding}

The current study was supported by the National Natural Science Foundation of China (grant no. 81441133) and the Natural Science Foundation of Hebei Province (grant no. H2013406096).

\section{Availability of data and materials}

The datasets used and/or analyzed during the current study are available from the corresponding author on reasonable request.

\section{Authors' contributions}

DL wrote the manuscript, performed the experiments and analyzed the data. CC and DW collected the data. ZC and CS conceived the idea, designed the study, provided technical support and revised the manuscript. All authors read and approved the final manuscript.

\section{Ethics approval and consent to participate}

All animal experimental procedures were approved by the Ethics Committee of Chengde Medical University.

\section{Patient consent for publication}

Not applicable.

\section{Competing interests}

The authors declare that they have no competing interests.

\section{References}

1. He Y, Zhang L, Fang Z, Jiang H, Liu X and Sun J: Regulation of Qizhen Jiangtang Granules on TGF- $\beta 1 /$ Smad signaling pathway in rats with diabetic nephropathy. $\mathbf{J}$ Anhui Univ Tradit Chin Med 39: 50-55, 2020.

2. Zhang J: The effect of calcium dobesilate capsule combined with ramipril in the treatment of early diabetic nephropathy. Henan Medical Res 28: 2020-2021, 2019.

3. Chen X and Fang M: Oxidative stress mediated mitochondrial damage plays roles in pathogenesis of diabetic nephropathy rat. Eur Rev Med Pharmacol Sci 22: 5248-5254, 2018.

4. Manna K, Mishra S, Saha M, Mahapatra S, Saha C, Yenge G, Nilesh Gaikwad, Pal R, Oulkar D, Banerjee K and Das Saha K: Amelioration of diabetic nephropathy using pomegranate peel extract-stabilized gold nanoparticles: Assessment of NF- $\mathrm{kB}$ and Nrf2 signaling system. Int J Nanomedicine 7: 1753-1777, 2019.

5. Sharma D, Bhattacharya P, Kalia K and Tiwari V: Diabetic nephropathy: New insights into established therapeutic paradigms and novel molecular targets. Diabetes Res Clin Pract 128: 91-108, 2017. 
6. Cheng YX, Yang LL and Lin Y: Research progress on the relationship between p38MAPK signaling pathway and glomerular disease. Shandong Medical J 58: 98-101, 2018.

7. Shaw PJ, McDermott MF and Kanneganti TD: Inflammasomes and autoimmunity. Trends Mol Med 17: 57-64, 2011.

8. Xuan HY, Chen YY and Wang CL: Inflammatory bodies and kidney diseases. J Nephrol Dial Ren Transpl 27: 369-373, 2018.

9. Guo Y, Song Z, Zhou M, Yang Y, Zhao Y, Liu B and Zhang X: Infiltrating macrophages in diabetic nephropathy promote podocytes apoptosis via TNF- $\alpha$-ROS-p38MAPK pathway. Oncotarget 8: 53276-53287, 2017.

10. Wang X, Shi L, Han Z and Liu B: Follistatin-like 3 suppresses cell proliferation and fibronectin expression via p38MAPK pathway in rat mesangial cells cultured under high glucose. Int J Clin Exp Med 8: 15214-15221, 2015.

11. Luo B, Li B, Wang W, Liu X, Liu X, Xia Y, Zhang C, Zhang Y, Zhang $\mathrm{M}$ and An F: Rosuvastatin alleviates diabetic cardiomyopathy by inhibiting NLRP3 inflammasome and MAPK pathways in a type 2 diabetes rat model. Cardiovasc Drugs Ther 28: 33-43, 2014

12. Xueyun $X$ and Hanli C: The clinical effect of Yiqi Huoxue Tongmai Decoction in adjuvant treatment of type 2 diabetic peripheral neuropathy. Inner Mongolia Tradit Chin Med 39: 9-11, 2020.

13. Lu M: Evaluation of the efficacy and drug side effects of Baitangping and metformin in the treatment of type 2 diabetes World Latest Medical Information Abstracts 18: 100-101, 2018.

14. Wang Q and Chang J: Subcutaneous injection of insulin causes local skin adverse reactions. Chin J Dermatol 48: 65-67, 2015.

15. Fu XM,Zhong MR and Fu WL: Effect of sericin on blood glucose and blood lipid in type 2 diabetic rats. China J Gerontol 31: 103-105, 2011

16. Song YX, Wang DD and Li DZ: Effect of sericin on the expression of extracellular signal-regulated kinase in the kidney of type 2 diabetic rats. China J Gerontol 36: 4050-4053, 2019.

17. Gao Y, Zhang M, Wu T, Xu M, Cai $\mathrm{H}$ and Zhang Z: Effects of D-Pinitol on insulin resistance through PI3K/Akt signaling pathway in type 2 diabetes mellitus rats. J Agric Food Chem 63: 6019-6026, 2015.

18. Yin M, Xu S, Wang Y, Sun X, Liang C, Li J and Mu Y: Changes in the Wnt/ $\beta$-catenin signaling pathway of the aorta of type 2 diabetic rats and the regulatory effect of SIRT1. Chin Pharmacol Bull 32: 337-342, 2016.

19. Zhang L, Wang L, Jia AO and Jia L: Effect of water extract of okra on glucose and lipid metabolism in type 2 diabetic rats. Sci Technol Food Ind 37: 355-358, 363, 2016.

20. Chen S, Liang F and Wang H: High-fat, low-sugar and high-fat, high-sugar diet to establish a rat model of impaired glucose tolerance. Chin J Gerontol 38: 1930-1932, 2018 (In Chinese).

21. Hao W, Li J and Jiang H: Advantages of purified ingredient high-fat/high-sugar feed and its domestic application status. J Hyg Res 46: 143-147, 2017.

22. You L, Lu K and Li Y: Intervention of triptolide on the expression of glucose transporter type 1 and type 4 in the myocardium of diabetic rats. J Prac Med Tech 22: 573-575, 2015.

23. Dong Y: Effects of changes in serum T, testicular ABP and INHB expression levels in hyperlipidemia and diabetic rats on spermatogenic function, respectively. Guangxi Medical University, Guangxi, 2018.

24. Zeng Z: The effect of telmisartan on the PERK/ATF4/CHOP pathway of endoplasmic reticulum stress in rats with diabetic myocardial damage. North China University of Technology, Hebei, 2018.

25. Livak KJ and Schmittgen TD: Analysis of relative gene expression data using real-time quantitative PCR and the 2(-Delta Delta $\mathrm{C}(\mathrm{T})$ ) method. Methods 25: 402-408, 2001

26. Yang D, Chen H, Liu Z, Chen H and Li Y: Study on the correlation between cystatin $\mathrm{C}$, retinol binding protein, free fatty acid and the occurrence and development of type 2 diabetic nephropathy. Mark Immunoassay Clin 27: 1022-1025, 1032, 2020.

27. Guang $S$, Zhai $R$ and Wang $L$ : The value of serum homocysteine, cystatin C, and superoxide dismutase in the early diagnosis of type 2 diabetic nephropathy. Chin J Lab Diagn 24: 1114-1117, 2020.

28. Xie R, Zhang HP and Liu XH: PINCH-1 protein and diabetic nephropathy. Chin J Clin 10: 2933-2936, 2016.

29. Yang W, Lu J, Weng J, Jia W, Ji L, Xiao J, Shan Z, Liu J, Tian H, Ji Q, et al: Prevalence of diabetes among men and women in China. N Engl J Med 362: 1090-1101, 2010

30. Guo L, Meng H and Gao A: Changes and significance of inflammatory factors and oxidative stress indicators in patients with diabetic nephropathy in different stages. J Inner Mongolia Med Univ 41: 370-373, 2019.
31. Wang Y, Yang L and Cheng T: Inflammatory pathogenesis of diabetic nephropathy and prevention and treatment of traditional Chinese medicine. Chin J Exp Formulas 24: 200-207, 2018.

32. O'Neil JD, Ammit AJ and Clark AR: MAPK p38 regulates inflammatory gene expression via tristetraprolin: Doing good by stealth. Int J Biochem Cell Biol 94: 6-9, 2018.

33. Liu Z, Lv Y, Zhang Y, Liu F, Zhu L, Pan S, Qiu C, Guo Y, Yang T and Wang J: Matrine-type alkaloids inhibit advanced glycation end products induced reactive oxygen species-mediated apoptosis of aortic endothelial cells in vivo and in vitro by targeting MKK3 and p38MAPK signaling. J Am Heart Assoc 6: e007441, 2017.

34. Chen DQ, Cao G, Chen H, Liu D, Su W, Yu XY, Vaziri ND, Liu XH, Bai X, Zhang L and Zhao YY: Gene and protein expressions and metabolomics exhibit activated redox signaling and $w n t / \beta$-catenin pathway are associated with metabolite dysfunction in patients with chronic kidney disease. Redox Biol 12: 505-521, 2017.

35. Zhang Y, Lu SL and Mo ML: Effects of expression of NF- $\kappa B$, NLRP3 inflammatory bodies and apoptotic factors in rats with diabetic nephropathy. Chin Tradit Med 16: 35-36, 2018.

36. Nitta T, Kanoh H, Inamori KI, Suzuki A, Takahashi T and Inokuchi JI: Globo-series glycosphingolipids enhance Toll-like receptor 4-mediated inflammation and play a pathophysiological role in diabetic nephropathy. Glycobiology 29: 260-268, 2019.

37. Pan XY, Gong XH and Shen FX: Effects of high glucose environment on the expression of IL-18, IL-1 $\beta$, IL-6, TNF- $\alpha$ in glomerular mesangial cells of HBZY-1 rats. China Integrat Tradit Chin Western Med J Nephrol 13: 519-521, 2012.

38. Miyamoto S, Shikata K, Miyasaka K, Okada S, Sasaki M, Kodera R, Hirota D, Kajitani N, Takatsuka T, Kataoka HU, et al: Cholecystokinin plays a novel protective role in diabetic kidney through anti-inflammatory actions on macrophage: Anti-inflammatory effect of cholecystokinin. Diabetes 61: 897-907, 2012.

39. He J, Chen X, Li B, Zhou W, Xiao J, He K, Zhang J and Xiang G: Chaetocin induces cell cycle arrest and apoptosis by regulating the ROS-mediated ASK-1/JNK signaling pathways. Oncol Rep 38: 2489-2497, 2017.

40. Yan Z, Liu S and Yan H: Research progress on the role of NLRP3 inflammasome in diabetic nephropathy. Shandong Med 57: 107-109, 2017.

41. Wang Z, Meng S, Cao L, Chen Y, Zuo Z and Peng S: Critical role of NLRP3-caspase-1 pathway in age-dependent isoflurane-induced microglial inflammatory response and cognitive impairment. J Neuroinflammation 15: 109-109, 2018.

42. Patel MN, Carroll RG, Galván-Peña S, Mills EL, Olden R, Triantafilou M, Wolf AI, Bryant CE, Triantafilou $\mathrm{K}$ and Masters SL: Inflammasome priming in sterile inflammatory disease. Trends Mol Med 23: 165-180, 2017.

43. Liu X, Zhang Z, Ruan J, Pan Y, Magupalli VG, Wu H and Lieberman J: Inflammasome-activated gasdermin $D$ causes pyroptosis by forming membrane pores. Nature 535: 153-158, 2016.

44. Jiang CX, Wu L and $\mathrm{Wu} \mathrm{J}$ : Curcumin analogs inhibit ERK/JNK and NF- $\mathrm{KB}$ signaling pathways and exert anti-inflammatory activities. Chin Tradit Herb Drugs 47: 2871-2876, 2016.

45. Zeng $\mathrm{S}$ and Zhou S: Research progress on diabetic nephropathy and oxidative stress related substances. Chin Med Eng 28: 37-40, 2020

46. Wang Z, Yang L and Meng X: Discussion on the research ideas of astragalus polysaccharides intervention in the pathogenesis of diabetic nephropathy. Chin Med Eng 33: 1-5, 2020.

47. LiY and Wang L: Research progress on inhibitors of sodium-glucose cotransporter 2. Med J Chinese People's Liberation Army: 9, 2019.

48. Zhou Y: Clinical efficacy of Bailing capsule combined with metformin in patients with diabetic nephropathy. Chin Tradit Patent Med: 2, 2019.

49. Zou Y: Advances in traditional Chinese and western medicine for diabetic nephropathy. Int J Urol 35: 154-156, 2015.

50. Li G, Zhang $\mathrm{H}$ and Li J: Research status of silkworm protein resources and its application in food industry. Food Ind Sci Technol 33: 396-401, 2012.

51. Li SZ: Compendium of Materia Medica. People's Medical Publishing House, Beijing, pp1561-1567, 1982.

52. Liu D, Wang X and Song C: Expression changes of liver tumor necrosis factor- $\alpha$ in type 2 diabetic rats and the protective effect of sericin. Chin J Gerontol 35: 6011-6013, 2015.

53. Li J, Liu D and Song C: Effect of sericin on insulin protein expression in islet cells of type 2 diabetic rats. Chin J Gerontol 34: 1549-1550, 2014

This work is licensed under a Creative Commons Attribution-NonCommercial-NoDerivatives 4.0 International (CC BY-NC-ND 4.0) License. 